\title{
A Multilevel-Multigrid Approach to Multiscale Electromagnetic Simulation
}

\author{
Peter Chow ${ }^{1}$, Tetsuyuki Kubota ${ }^{2}$, and Takefumi Namiki ${ }^{3}$ \\ ${ }^{1}$ Fujitsu Laboratories of Europe, \\ Hayes Park Central, Hayes, UB4 8FE, United Kingdom \\ peter.chow@uk.fujitsu. com \\ ${ }^{2}$ Corporate Component Engineering Center, Fujitsu Limited, \\ Nakahara-ku, Kawasaki 211-8588, Japan \\ t.kubota@jp.fujitsu.com \\ ${ }^{3}$ Computational Science and Engineering Solutions Center, Fujitsu Limited, \\ Chiba 261-8588, Japan \\ t.namiki@jp.fujitsu.com
}

\begin{abstract}
The time-dependent Maxwell's equations are solved for mobile device applications using a multilevel-multigrid finite-difference timedomain (FDTD) method. For three-dimensional models that simulate system level details of mobile devices, the smallest features are in the nanometre $\left(10^{-9} \mathrm{~m}\right)$ range, leading to a time-step size in the attosecond $\left(10^{-18} \mathrm{~s}\right)$ range. The feature sizes of mobile devices are in the centimetre $\left(10^{-2} \mathrm{~m}\right)$ range, while for health and safety studies that include human models features are in the metre range.
\end{abstract}

\section{Introduction}

The drive towards compact, lightweight and fast mobile electronic devices has fuelled the demand for electromagnetic simulations/analyses that employ complete models in fine detail but at the same time show shortened simulation times. In general, detailed models and faster simulations are not compatible - detailed models by nature are associated with long computational times. The most popular method for computational electromagnetic simulation, the finite-difference time-domain (FDTD) method [1,2], is no different. The FDTD method solves the time-dependent Maxwell's equations using the explicit leapfrog time-stepping scheme. The Courant-Friedrichs-Lewy (CFL) stability condition restricts the size of the time-step directly to the smallest feature in the domain. For threedimensional models that simulate system level details of mobile devices, the smallest features are in the nanometre $\left(10^{-9} \mathrm{~m}\right)$ range, leading to a time-step size in the attosecond $\left(10^{-18} \mathrm{~s}\right)$ range. The feature sizes of mobile devices are in the centimetre $\left(10^{-2} \mathrm{~m}\right)$ range, while for health and safety studies that include human models features are in the metre range. Direct analysis of such models with the FDTD method is extremely demanding in both memory and computer time. Here, we apply the multilevel-multigrid grid embedding approach to address such multiscale simulations in both spatial and temporal spaces. We also 
introduce the block-multigrid concept which makes the multigrid FDTD method even more efficient.

\section{Multilevel-multigrid FDTD Method}

In this section, the multilevel-multigrid FDTD method is introduced one element at a time for clarity, with references being given to more detailed explanations. First the multigrid FDTD method is introduced, followed by the block-solve enhancement, and finally the multilevel element is discussed.

\section{$2.1 \quad$ Multigrid FDTD}

The multigrid FDTD method [3-9] solves Maxwell's equations based on the finitedifference time-domain (FDTD) method [1,2] for the calculation of electromagnetic wave propagation by strategically embedding finer grids in regions that require high resolution and not everywhere. Fig. 1]illustrates the grids of normal FDTD and multigrid FDTD methods. The multigrid approach greatly reduces memory and computational cost compared with an FDTD method of similar accuracy. Furthermore, the Cartesian grid used in the finite-difference scheme is considered the most efficient in terms of memory requirement and computational efficiency.

\subsection{Block-Solve Multigrid FDTD}

For most calculations the multigrid FDTD method is fast and efficient. However, there are situations where the embedded Cartesian grid is very inefficient, such as elongated irregular signal-line type structures. Owing to the rectangular nature of Cartesian grids, they can cover large empty spaces. Fig. 2 shows such a model where the embedded grid (the rectangle covering the signal-line structure) is covering some 50 per cent of empty signal-line space as illustrated by the two dotted-line ovals. Naturally, removing such empty space will reduce memory
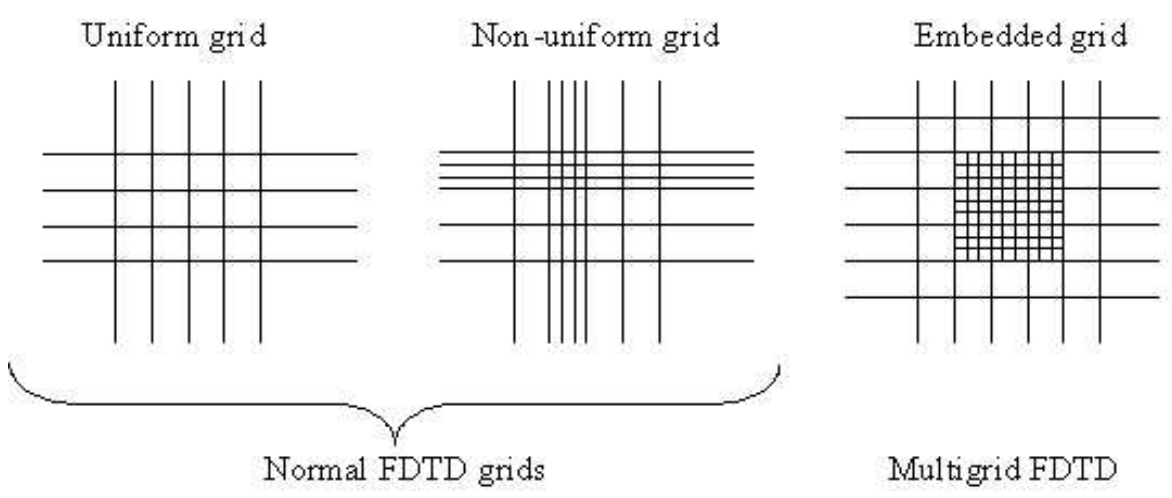

Fig. 1. Comparison of grid types used in FDTD and multigrid FDTD methods 


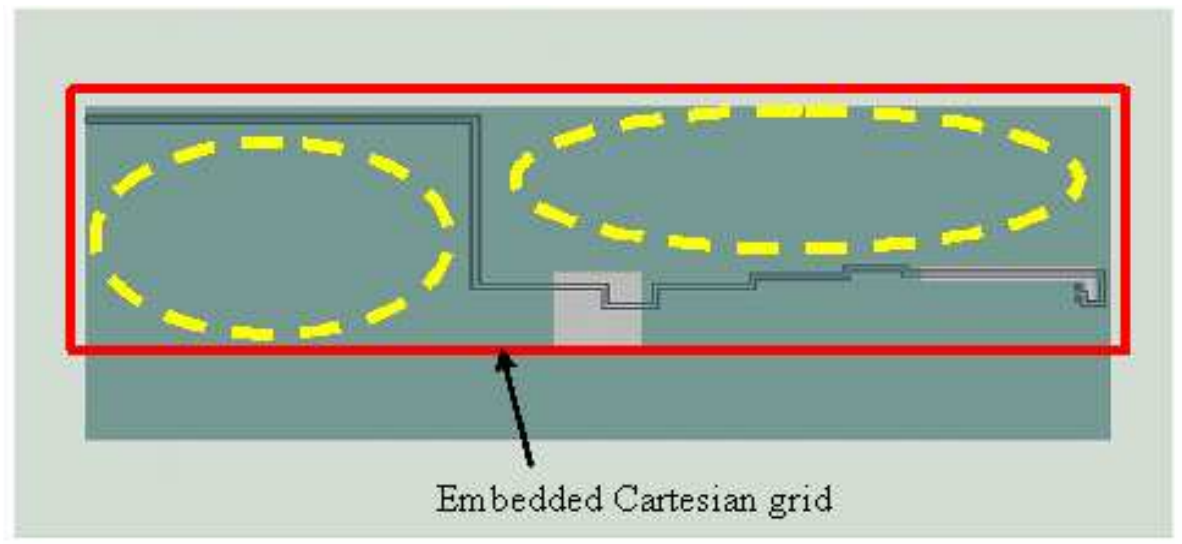

Fig. 2. Embedded grid covering an irregular signal-line structure

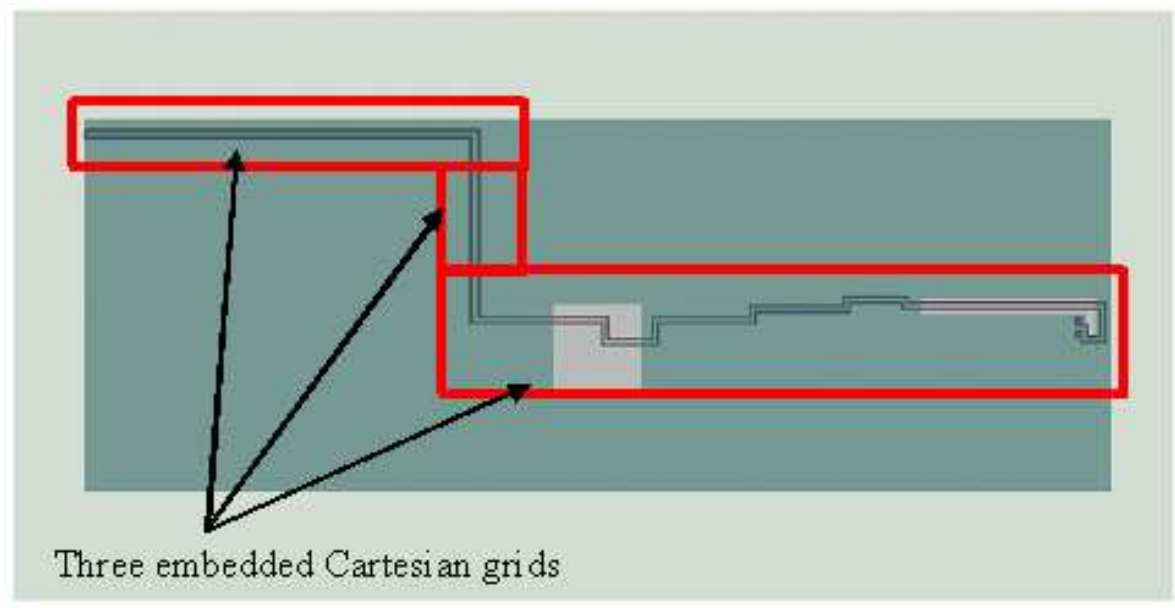

Fig. 3. Multiple Cartesian grids covering the signal-line structure

requirements and the number of calculations thus shortening the solution time. Fig. 3 shows a configuration of three embedded grids covering the signal-line structure.

Applying the multigrid FDTD method directly to the model in Fig. 3 will give an incorrect solution. This is because the temporal space at the adjoining interface between the grids in the fine-grid time-step level is not directly communicated. Instead, it is via the coarse grid where it is interpolated with the rest of the boundary from coarse-grid values. Therefore, a modification to the solution procedure to include the exchange of values between adjoining grids at each embedded time-step level is required. The block-solve solution procedure for multigrid FDTD is detailed in [10] and will not be repeated here. 


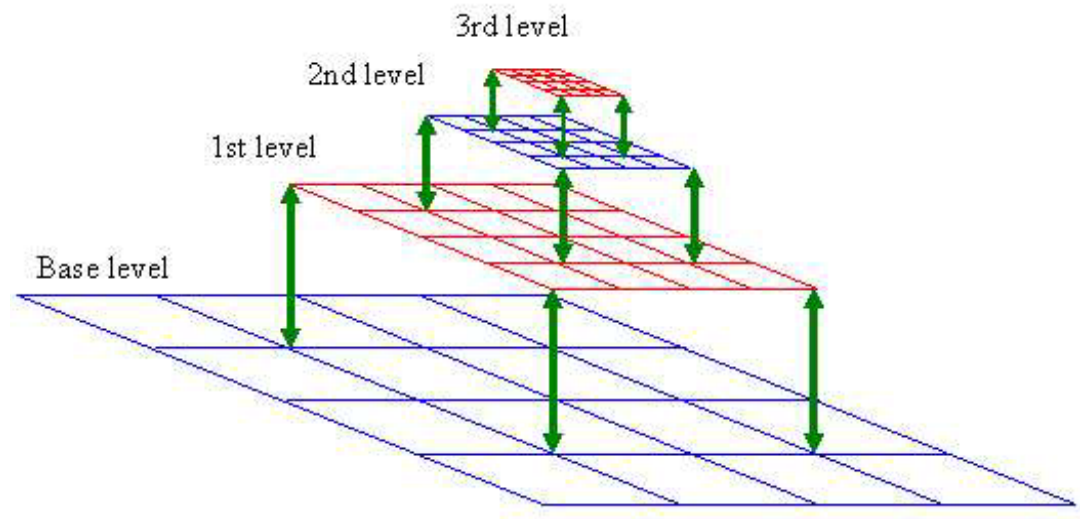

Fig. 4. Multi-level grid configuration

\section{$2.3 \quad$ Multilevel-multigrid FDTD}

Fig. 4 shows a multilevel grid configuration (in two dimensions) of three levels of grid embedding, with each embedded grid finer than the one before. The multilevel-multigrid FDTD method [11] provides a refinement process where the grid cell aspect scale changes gradually as opposed to a severe change. This refinement applies to both spatial and temporal spaces. Thus, a gradual refinement is commonly a more stable approach than a severe change all at once.

\section{Example Model and Results}

Fig. [5] shows a simple example model with geometric scale aspect ratio of about 1,000 between the smallest feature in the model and the largest. The smallest feature is the coil wire width of 1 micrometre $\left(10^{-6} \mathrm{~m}\right)$ and the largest component is the strip-line with a length in the millimetre range.

Table 1 shows five grid models and their respective results. The first two grid models, coarse and fine grids, are for the standard FDTD method. The fine grid model is created by refining the coarse grid with a refinement factor of 2 . The coarse grid model has one cell for the width of the coil wire, and the fine grid has four $(2 \times 2)$ cells due to the refinement of 2 . The respective time-step sizes calculated from the CFL stability condition are 2.07 and 1.035 picoseconds $\left(10^{-15} \mathrm{~s}\right)$.

The other grid models are for the multilevel-multigrid FDTD method and a refinement of 2 is applied at each grid level. The third grid model (MG 1level) has one level of embedded grid, so the base grid has the same time-step size as the coarse grid model. The fourth grid model (MG 4-levels) has four levels of grid refinement at the coil region (giving one cell for the width of the coil wire) and one level at the port regions. The final grid model (MG 5levels) has five levels of grid refinement at the coil region, giving four $(2 \times 2)$ cells 


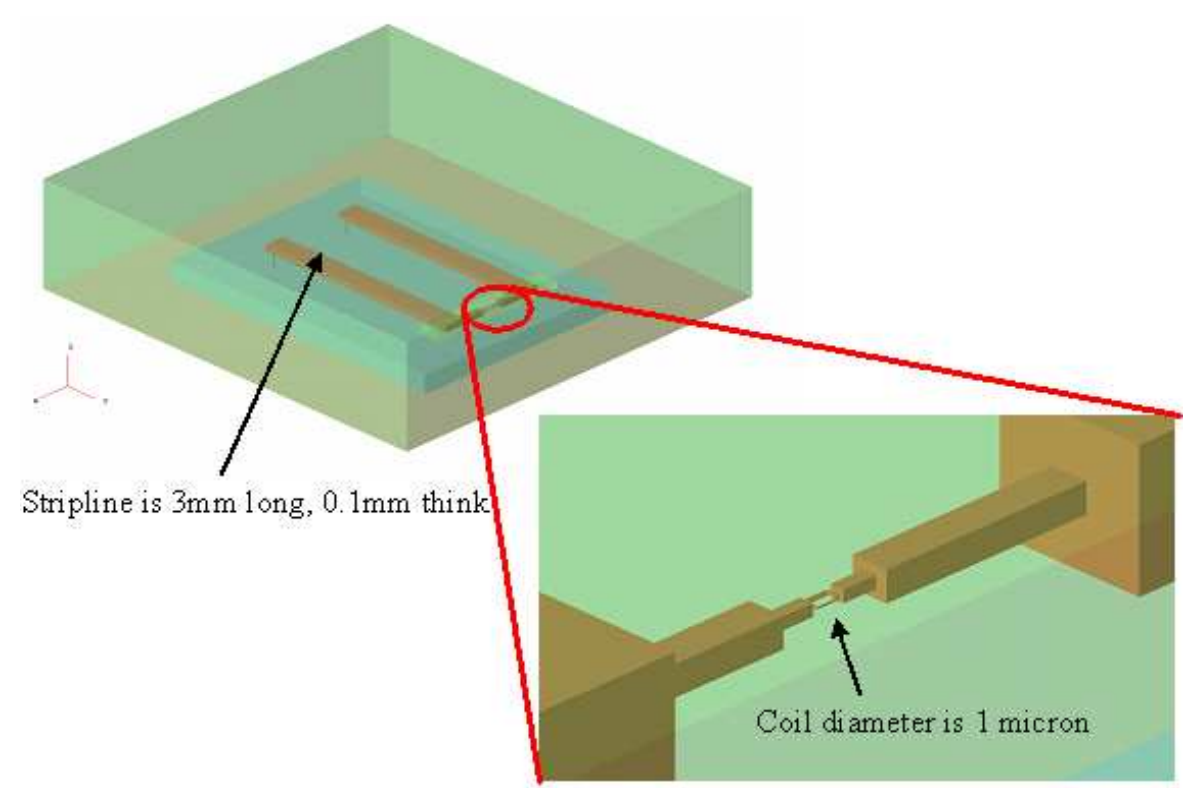

Fig. 5. Example model

Table 1. Computing time, grid levels, and size og grids

\begin{tabular}{|c|c|c|c|c|}
\hline Model & $\begin{array}{r}\mathrm{CPU} \\
\text { seconds }\end{array}$ & Grid size & Speedup & Speedup \\
\hline Coarse grid & 3,402 & $63 \times 60 \times 50$ & $1 \times 1$ cell & \\
\hline Fine grid & 47,954 & $125 \times 119 \times 99$ & & $2 \times 2$ cells \\
\hline $\begin{array}{l}\text { MG 1-level } \\
(2 \times 2 \text { cells width of coil })\end{array}$ & 11,655 & $\begin{array}{l}63 \times 60 \times 50 \text { base } \\
50 \times 52 \times 56\end{array}$ & & 4.1 \\
\hline $\begin{array}{l}\text { MG 4-levels } \\
(1 \times 1 \text { cell coil centre })\end{array}$ & 1,577 & $\begin{array}{l}46 \times 44 \times 28 \text { base } \\
32 \times 32 \times 26 \text { level-1 } \\
47 \times 35 \times 35 \text { level- } 2 \\
43 \times 27 \times 31 \text { level-3 } \\
35 \times 35 \times 35 \text { level- } 4\end{array}$ & 2.16 & \\
\hline MG 1-level at each port & & $\begin{array}{l}19 \times 17 \times 31 \text { port- } 1 \\
19 \times 17 \times 31 \text { port- } 2\end{array}$ & & \\
\hline $\begin{array}{l}\text { MG } 5 \text {-levels } \\
(2 \times 2 \text { cells coil centre })\end{array}$ & 10,028 & $\begin{array}{l}46 \times 44 \times 28 \text { base } \\
32 \times 32 \times 26 \text { level-1 } \\
47 \times 35 \times 35 \text { level- } 2 \\
51 \times 43 \times 43 \text { level- } 3 \\
63 \times 59 \times 57 \text { level- } 4 \\
39 \times 55 \times 55 \text { level- } 5\end{array}$ & & 4.78 \\
\hline MG 1-level at each port & & same size as before & & \\
\hline
\end{tabular}




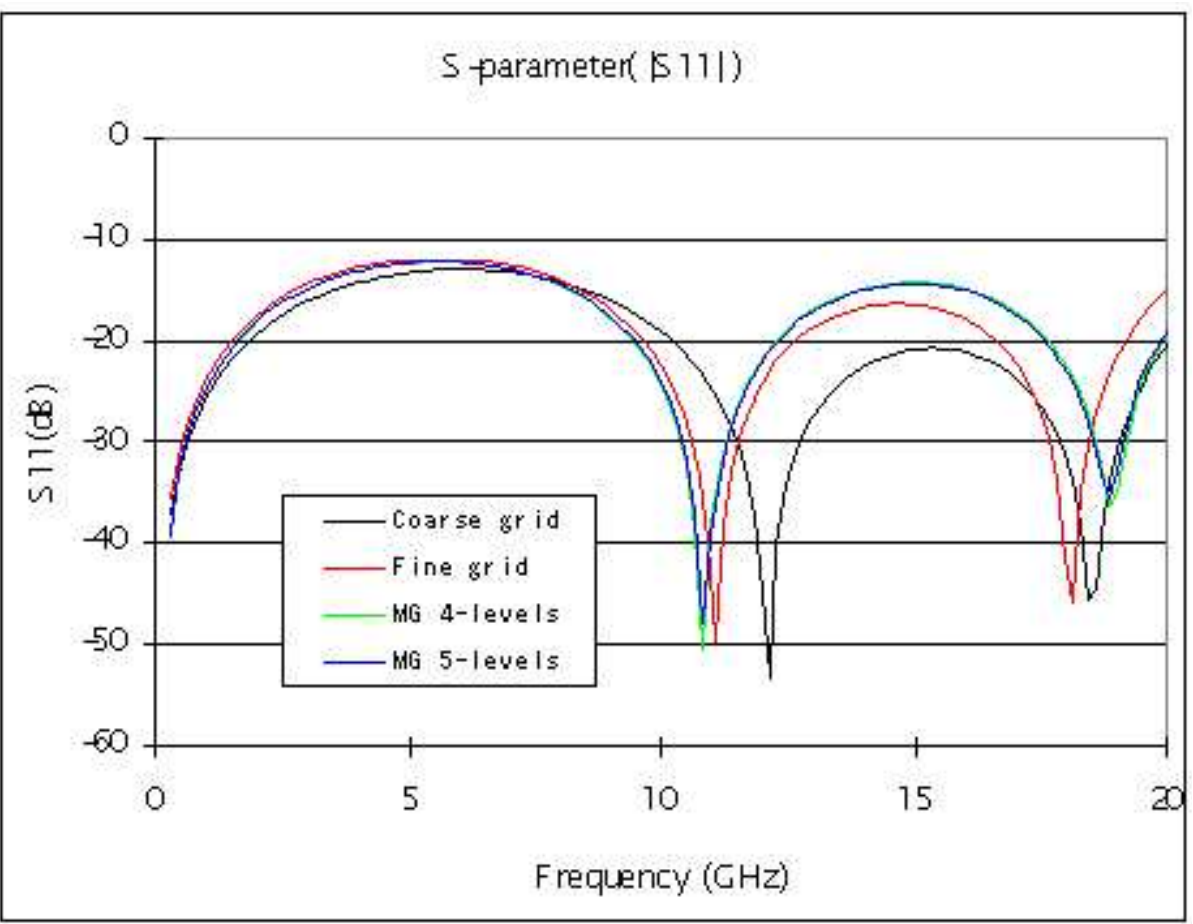

Fig. 6. Plot of S-parameters

for the width of the coil wire and, as for the fourth model, one level at the port regions. The last two grid models both have a base-grid time-step of 33.56 picoseconds.

The 1-level embedded grid model attains a speedup of 4.1 compared with the fine grid model, whereas the 5-levels model attains a 4.78 speedup. The 4 -level embedded grid model attains a 2.16 speedup compared with the coarse grid model.

Fig. 6] shows the S-parameter for all the grid models. There is good agreement between the fine grid and multilevel-multigrid FDTD methods up to 15 $\mathrm{GHz}$, with slight deviations starting to appear above $15 \mathrm{GHz}$. Also, the plot shows that the coarse grid result has the worst result due to insufficient grid resolution.

\section{Conclusions}

We have used a multilevel-multigrid approach to address multiscale problems for three-dimensional electromagnetic wave propagation. The result obtained for features in the micrometre $\left(10^{-6}\right)$ range is encouraging. The challenge now is to advance the technique to the nanometre $\left(10^{-9}\right)$ range. 


\section{References}

1. Yee, K. S.: Numerical Solution of Initial Boundary Value Problems Involving Maxwell's Equations in Isotropic Media. IEEE Trans. Antennas Propagation 14 (1996) 302-307

2. Taflove, A.: Computational Electrodynamics: The Finite-Difference Time-Domain Method. Artech House, Boston (1995)

3. Kim, I.S., Hoefer, W.J.R.: A Local Mesh Refinement Algorithm for the Time Domain Finite Difference Method Using Maxwell's Curl Equations. IEEE Trans. Microwave Theory Tech. 38 (1990) 812-815

4. Zivanovic, S.S., Yee, K.S., Mei, K.K.: A Subgridding Method for the Time-Domain Finite-Difference Method to Solve Maxwell's Equations. IEEE Trans. Microwave Theory Tech. 39 (1991) 471-479

5. Monk, P.: Sub-Gridding FDTD Schemes. J. Applied Computational Electromagnetic Society 11 (1996) 37-46

6. Thomas, P., Weiland, T.: A Consistent Subgridding Scheme for the Finite Difference Time Domain Method. Int. J. Numerical Modelling: Electronic Networks, Devices \& Fields 9 (1996) 359-374

7. Chevalier, M.W., Luebbers, R.J., Cable, V.P.: FDTD Local Grid with Material Traverse. IEEE Trans. Antennas Propagation 45 (1997) 411-421

8. Okoniewski, M., Okoniewska, E., Stuchly, M.A.: Three-Dimensional Subgridding Algorithm for FDTD. IEEE Trans. Antennas Propagation 45 (1997) 422-429

9. White, M.J., Yun, Z., Iskander, M.F.: A New 3D FDTD Multigrid Technique with Dielectric Traverse Capabilities. IEEE Trans. Microwave Theory Tech. 49 (2001) 422-430

10. Chow, P., Kubota, T., Namiki, T.: A Block-Solve Multigrid-FDTD Method. To appear in the 22nd International Review of Progress in Applied Computational Electromagnetics (ACES 2006) conference in March 2006, Miami, Florida, USA

11. Chaillou, S., Wiart, J., Tabbara, W.: A Subgridding Scheme Based on Mesh Nesting for FDTD Method. Microwave and Optical Technology Letters 22 (1999) 211-214 